\title{
Contribuições da Análise Conversacional ao estudo do traumatismo craneo-encefálico: relato de um caso
}

\section{Contributions of Conversation Analysis to the study of traumatic brain injury: a single case report}

\author{
Véronique Agnes Guernet Steiner ${ }^{1}$, Letícia Lessa Mansur ${ }^{2}$
}

\begin{abstract}
RESUMO
A persistência de dificuldades no processo de comunicação em sujeitos acometidos por um traumatismo craneo-encefálico requer a utilização de instrumentos de avaliação, sensíveis às alterações lingüístico-cognitivas apresentadas, que possam facilitar a reintegração desses indivíduos na sociedade. O presente estudo de caso investiga as contribuições da Análise Conversacional, na avaliação das habilidades funcionais de um jovem de 27 anos, acometido por um traumatismo craneo-encefálico grave. Analisa uma interação conversacional espontânea por meio do estudo do mecanismo colaborativo da tomada de turno, do gerenciamento do tópico e das reformulações, comparando os resultados com testes formais de linguagem. Discute as contribuições dessa abordagem ao processo de reabilitação fonoaudiológica. Enquanto os testes formais não indicaram alterações de linguagem, a Análise Conversacional permitiu, ao analisar a seqüência de turnos e as reações mútuas dos interlocutores, identificar problemas comunicativos e verificar como os interlocutores lidavam com os mesmos. A Análise Conversacional mostrou-se sensível às alterações lingüístico-cognitivas apresentadas pelo traumatismo craneo-encefálico, fornecendo elementos concretos para, no processo de reabilitação, abordar, de forma organizada, o discurso mais utilizado no cotidiano.
\end{abstract}

DESCRITORES: Traumatismos cerebrais; Transtornos da comunicação; Testes de linguagem; Reabilitação; Patologia da fala e linguagem; Relatos de casos [Tipo de publicação]

\section{INTRODUÇÃO}

A persistência das alterações lingüístico-cognitivas em sujeitos acometidos pelo traumatismo craneo-encefálico (TCE) é, atualmente, considerada como um problema sério para a reinserção desses indivíduos na sociedade ${ }^{(1)}$. O sucesso social, vocacional, familiar e acadêmico depende de uma função comunicativa efetiva destes sujeitos. Apesar de, freqüentemente, não apresentarem dificuldades na realização de tarefas das provas formais linguagem, têm dificuldades no uso da linguagem, ou seja, na adaptação da linguagem e da comunicação às demandas sociais específicas. A inserção desses indivíduos em um processo de reabilitação fonoau-

(1)Pós-graduanda do Programa de Pós-graduação em Ciências da Reabilitação - Comunicação Humana do Departamento de Fisioterapia, Fonoaudiologia e Terapia Ocupacional da Universidade de São Paulo - USP - São Paulo (SP), Brasil.

(2) Professora do Curso de Fonoaudiologia da Universidade de São Paulo - USP - São Paulo (SP), Brasil; Responsável pelo Laboratório de Investigação em Neurolingüística do Departamento de Fisioterapia, Fonoaudiologia e Terapia Ocupacional da Universidade de São Paulo - USP - São Paulo (SP), Brasil.

Endereço para correspondência: Véronique Steiner. Av. IV Centenário, 1578, São Paulo - SP, CEP 04030-000. E-mail: vsteiner@uol.com.br Recebido em: 5/7/2007; Aceito em: 8/2/2008 diológica depende da utilização de instrumentos específicos de avaliação que sejam sensíveis às alterações encontradas e que possam fornecer elementos concretos para o processo de reabilitação.

No enfoque pragmático, analisa-se o discurso para apreciar o uso da linguagem, particularmente os mecanismos adaptativos empregados pelos interlocutores em determinado contexto espacial e temporal ${ }^{(2)}$. Como o processamento do discurso envolve a interação entre conhecimento lingüístico e habilidades cognitivas (incluem, para mencionar apenas algumas, memória de trabalho, planejamento, resolução de problemas, atenção e funções executivas), seu estudo permite o estudo da interface cognitivo-comunicativa no TCE. Estudos recentes buscam compreender essa complexa interação para avaliar e desenvolver intervenção a longo-prazo das alterações da função comunicativa dos sujeitos com TCE, inclusive em relação ao discurso mais utilizado no dia-a-dia, a conversação ${ }^{(3-4)}$.

O desenvolvimento de uma atividade dialógica compreende vários mecanismos de regulação/ monitoração, solicitando, portanto, a integridade e flexibilidade dos processos cognitivos. Não é raro observar, nos casos de TCE, dificuldades com a tomada de turnos, com a iniciação, desenvolvimento e manutenção dos tópicos, com a flexibilidade necessária para reformular um enunciado e para utilizar-se de estratégias 
compensatórias adequadas. Para uma revisão ver Mansur LL, Radanovic $\mathrm{M}^{*}$.

A conversação tem sido estudada por meio de duas abordagens na literatura da área de neuropsicologia ${ }^{(5)}$. A primeira, "top-down", provém da lingüística e da filosofia da linguagem. Utiliza-se de um raciocínio dedutivo, no qual uma competência abstrata serve como modelo para enunciadores e situações ideais. Valoriza a observação da utilização das máximas conversacionais, o estudo da relevância e dos atos de fala. A segunda, "bottom-up", provém da etnometodologia. Não parte de princípios analíticos ou organizacionais, mas examina indutivamente a conversação em situações naturais para, a seguir, descrever os mecanismos colaborativos que os interlocutores utilizam. Utiliza-se do arcabouço teórico da Análise Conversacional (AC) para descrever como os interlocutores revelam intersubjetivamente as interpretações das falas respectivas ${ }^{(6)}$. A AC foi adotada como instrumento de avaliação para lesados cerebrais afásicos ${ }^{(7)} \mathrm{e}$, também, para indivíduos com alterações $\operatorname{cognitivas}^{(8)}$. Foi descrita como instrumento sensível para a identificação e investigação dos déficits pragmáticos no $\mathrm{TCE}^{(9)}$. Em nosso país, a AC foi estudada principalmente em discursos de indivíduos normais ${ }^{(10)}$

O presente artigo pretende verificar as contribuições da $\mathrm{AC}$ ao estudo das alterações da função comunicativa no TCE, ilustrando o uso dessa abordagem com um estudo de caso. Discute as contribuições da AC na investigação das habilidades conversacionais e suas implicações para o processo de reabilitação.

\section{A Análise Conversacional}

A AC parte do pressuposto que as conversações são organizadas, tendo como unidade de análise o turno, definido como "a contribuição conversacional de um enunciador, seguida de um silêncio ou de uma contribuição do interlocutor"(11). Valoriza, na maioria dos estudos, principalmente a análise dos mecanismos de tomada de turno, o gerenciamento do tópico e os mecanismos de reformulação.

A AC analisa as conversações em um contexto ecológico, pois estuda conversações espontâneas em contextos naturais, evitando qualquer tipo de artificialidade no estabelecimento da interação. Não inclui a análise das intenções comunicativas ou a categorização dos enunciados, segundo sua adequação comunicativa. Permite, portanto, o desenvolvimento de intervenção muito próxima da realidade vivenciada pelos interlocutores no cotidiano.

A AC trata a conversação como uma realização colaborativa, de responsabilidade conjunta de ambos os interlocutores. A interação é mais do que a soma das atividades dos participantes; cada intervenção feita por um interlocutor revela a análise que ele fez do turno anterior e constitui, ao mesmo tempo, o contexto para o turno subseqüente. Qualquer ocorrência na sequiência de turnos (até pausas e sobreposições de fala) é incluída na análise, pois pode, potencialmente, agir sobre o turno subseqüente.
Vale notar que as conversas espontâneas em contextos naturais sempre apresentam um número significativo de pausas, frases não terminadas e violações de turno que são, geralmente, resolvidas pelos interlocutores. No TCE, no entanto, essas falhas podem vir a ser problemáticas.

\section{APRESENTAÇÃO DO CASO CLÍNICO}

\section{Descrição do sujeito}

IP tem 27 anos, gênero masculino, solteiro, tendo concluído o $3^{\circ}$ grau na área de engenharia. Sofreu um traumatismo craneo-encefálico grave decorrente de acidente de carro, com pontuação inicial de 06 na Escala de Glasgow. Ele ficou dois meses em coma e procurou o setor de Fonoaudiologia 22 meses após o acidente.

O laudo da primeira ressonância magnética indica múltiplas lesões encefálicas, a maioria com componente hemorrágico e associação de lesão axonal difusa. Dez meses após o acidente foi submetido à SPECT, que revelou hipoperfusão focal acentuada em região frontal direita.

Apresentou evolução positiva na função comunicativa, chegando a retomar, 14 meses após o acidente, a mesma atividade profissional de engenheiro civil exercida antes do acidente. Apesar de não apresentar nenhuma seqüela do ponto de vista físico ou locomotor, IP relata que sua vida mudou muito após o acidente. Sua queixa no momento da avaliação diz respeito a dificuldades para expressar suas idéias de forma clara e rápida. Evita situações que possam envolver discussão ou argumentação e refere que apresenta dificuldade para falar em grupo ou no telefone. Essas dificuldades interferem no seu desempenho profissional, causando-lhe "medo de perder o emprego" (SIC).

\section{Procedimento}

Além da avaliação da conversação por meio da AC, o sujeito foi submetido a uma anamnese fonoaudiológica, assim como aos seguintes testes formais: teste de Edinburgo ${ }^{(12)}$, Boston reduzido $^{(13)}$, Boston Naming ${ }^{(14)}$, Token Test ${ }^{(15)}$ versão reduzida, assim como um teste de fluência verbal semântica.

As avaliações foram aplicadas em três sessões. A análise da conversação baseou-se em dez minutos de conversação filmados, entre o sujeito (IP) e sua irmã (C). O tema da conversação foi introduzido por meio da leitura, feita pela examinadora, de uma notícia a respeito de "violência na cidade de São Paulo". A seguir, IP e sua irmã foram convidados a conversar sozinhos sobre o tema proposto. No intuito de obter amostra o mais próximo da situação espontânea, o pesquisador desempenhou papel de indutor de diálogo e, após alguns minutos, o temaalvo foi introduzido. Os minutos iniciais da interação não foram utilizados na análise. $\mathrm{O}$ trecho foi transcrito e analisado segundo o método da AC.

Três aspectos foram selecionados para a análise da conversação: a iniciação e tomada de turno, o gerenciamento do

* Mansur LL, Radanovic M. Alterações de linguagem nos traumatismos cranioencefálicos. In: Mansur LL, Radanovic M. Neurolingüística: Princípios para a prática clínica. São Paulo: Edições Inteligentes; 2004. p. 223-9. 
Quadro 1. Anamnese e resultados das avaliações formais

\begin{tabular}{|lll|}
\hline Exames realizados & Aspecto avaliado & Resultados \\
\hline Anamnese fonoaudiológica & Voz, fala deglutição & Sem alterações de voz fala ou deglutição \\
Teste de Edimburgo & Dominância manual & $100 \%$ (destro absoluto) \\
Boston reduzido & Compreensão oral: & $100 \%$ de acerto \\
& Produção oral & $100 \%$ de acerto \\
& Compreensão escrita & $100 \%$ de acerto \\
& Produção escrita & $100 \%$ de acerto \\
Teste de nomeação de Boston & Denominação de figuras & $95 \%$ de acerto \\
Teste Token & Compreensão oral & $100 \%$ de acerto \\
Fluência Verbal Semântica & Média de número de animais, frutas e itens do & 20 \\
& supermercado emitidos em 1 minuto & \\
\hline
\end{tabular}

tópico e as reformulações. Todos foram analisados segundo o grau de dificuldade na conversação (evidência na conversação) e segundo sua gravidade. Estes itens foram analisados segundo a(s) resposta(s) dada(s) pelo(s) interlocutor(es) na construção dos turnos.

A iniciação e a tomada de turno foram analisadas com relação à presença de pausas e distribuição dos turnos, no contexto da seqüência de contribuições efetivas e com relação ao mecanismo de partilha interativa do tempo.

O gerenciamento de tópico foi analisado segundo a iniciação, mudança e manutenção de tópico, na construção colaborativa da coerência na seqüência dos turnos.

$\mathrm{O}$ estudo das reformulações foi realizado verificando a trajetória das mesmas, desde a sua iniciação até a efetiva solução. Foram analisadas com relação às solicitações de reformulação por parte do interlocutor (hetero-reformulações) e com relação às reformulações realizadas sobre os próprios erros (auto-reformulações). O estudo inclui, além do estudo das reformulações propriamente ditas (repairs), o estudo dos "ensaios de formulação" (prepairs), seguindo a classificação das reformulações feita por Schlenck et al. ${ }^{(16)}$. Enquanto as primeiras, que chamaremos simplesmente de "reformulações", refletem a tentativa de corrigir os erros após sua articulação, os "ensaios de formulação" refletem um comportamento de busca que parece estar dirigido "à parte seguinte do enunciado", não sendo precedidos por um erro na fala externa.

\section{RESULTADOS}

\section{Anamnese e avaliações formais}

Na anamnese, conforme o Quadro 1, IP não relatou alterações de voz, fala ou da deglutição. Refere que não apresentou problemas na aquisição e desenvolvimento de linguagem. Também relata que não apresentava problemas com a função comunicativa antes do acidente, nem de fluência.

O teste de Edimburgo revelou dominância manual direita. Apresentou $100 \%$ de acerto no Boston reduzido para emissão e compreensão oral/gráfica, $95 \%$ de acerto no teste de nomeação de Boston. No Token Test, apresentou $100 \%$ de acerto. No teste de fluência verbal, emitiu 17 palavras para os nomes de animais, 18 palavras para a categoria de frutas e 24 palavras para a categoria de itens do supermercado, perfazendo uma média de 20 itens.

\section{Análise conversacional}

\section{Iniciação e tomada de turno}

IP apresenta um número muito elevado de pausas vazias, como pode ser observado no trecho 1, transcrito abaixo (para os símbolos utilizados na transcrição, vide apêndice). Chega a realizar, em um mesmo turno, duas pausas maiores do que um minuto como pode ser observado nos turnos 02 e 10 do trecho 1. Resulta em turnos longos com várias lacunas que induzem a interrupções por parte de seu interlocutor. Sua irmã constitui-se em um interlocutor privilegiado, pois respeitou a grande maioria das pausas produzidas por IP.

Vale notar que, apesar do número elevado de pausas, IP mantêm a atenção centrada e adere ao tópico introduzido, desenvolvendo-o segundo núcleo semântico-pragmático relevante, como pode ser observado na transcrição abaixo (trecho 1).

\section{Trecho 1}

01. C - cê não fica preocupado com a violência?

02. IP - eu fico um pouco (.) desde que eu::eu fui assaltado assim (1s.34ms.) antes eu:: não (.) antigamente eu não ligava tanto assim de sair de noi::te (.) lembra que eu saia (.) dormia fora né na ca/ na casa do Alexandre (.) eu num(.) eu num tinha tanto medo de sair (.) à noite (.) quanto eu tenho agora ( $1 \mathrm{~s} .27 \mathrm{~ms}$.) porque:: nunca é::: muito mais (.) é muito maior o índice de de de violência.

03. C - é cê ficou traumatizado.

04. IP - não não é traumatizado

05. C - cê não fica preocupado [quando chega alguém assim perto no vidro do carro?

06. IP [não isso aí não mudou num:: não mudou de como era antes e como é agora

07. C - Antes do seqüestro?

08. IP - é/ antes de:: é\#

09. C - \# do seu assalto?

10. IP - meio:: meia:: meia:: (1s.03ms.) susto assim né meu não mu/ 
não mudou com essas coisas né (1s.03ms.) mas:: o que:: eu percebo assim que mudou muito é:: esse problema de violência (.) que:: quando a gente tinha a idade do Arturzinho\#

11. C - \# a gente brincava na rua.

Com relação à distribuição dos turnos, observa-se que, sempre que IP foi selecionado como próximo interlocutor, respondeu prontamente às perguntas, tomando o turno sem demora. No entanto, em vários momentos, o fluxo conversacional foi interrompido pela irmã para solicitar um esclarecimento, como, por exemplo, nos turnos 08/09 e 10/11 do trecho 1 . Algumas vezes, IP não esclarece imediatamente a dúvida da irmã, o que leva a uma fragmentação do discurso, como ocorre no trecho abaixo (trecho 2).

\section{Trecho 2}

01. IP - aí eu fui andando bem devagar (.) aí::ch/ é (.) é tava na subida (.) é cum $(1 \mathrm{~s} .41 \mathrm{~ms}$.) quando chegou na curva (1s.07ms.) e fui fazendo a curva devagarinho aí fui vendo (.) a/ ai apareceu um cara fazendo a curva no maior pau.

02. $\mathrm{C}$ - veio de traz?

03. IP - por fora

04. C - veio lá de baixo?

05. IP - é

06. $\mathrm{C}$ - atrás de você?

07. IP - isso (.)veio no maior pau fazendo a curva por fora.

É interessante notar que IP não responde à primeira solicitação de esclarecimento de $\mathrm{C}$ nem percebe que suas respostas não esclarecem a dúvida de sua irmã. Duas explicações são possíveis: ou IP não percebe que há, neste momento, uma lacuna no conhecimento partilhado ou não consegue dirigir sua atenção para o aspecto a ser esclarecido, ou seja, saber de onde surgiu o carro do assaltante e adaptar/organizar a resposta de modo a atender a esse contexto específico.

Em vários momentos, os interlocutores violaram o turno do outro, chegando a haver sobreposição de vozes (turnos 05/06 do trecho 1). As violações de turno ocorreram tanto por parte de IP (sobreposição de vozes citada acima) quanto por parte de $\mathrm{C}$ (turnos 08/09 e 10/11 do trecho 1).

O Quadro 2, adaptado do manual de aplicação do CAPPCI, resume as habilidades e dificuldades de IP com relação à iniciação e tomada de turno, e seu impacto na conversação.

\section{Gerenciamento do tópico}

Ambos os interlocutores iniciam tópicos. Apesar das dificuldades de formulação, ambos os parceiros conseguem desenvolver sequencialmente vários turnos a respeito da violência. No entanto, vários problemas surgiram na coerência tópica quando as dificuldades de formulação de IP levaram sua irmã a solicitar esclarecimentos, chegando a haver o abandono de um subtópico. No trecho 1, por exemplo, C introduz um tópico a respeito da sua preocupação sobre o aumento da violência (turno 01) ao qual IP responde no turno 02. No entanto, quando IP tenta introduzir um subtópico no turno 06, apresenta dificuldades de formulação que levam à solicitação de esclarecimento por parte de $\mathrm{C}$ nos turnos $07 \mathrm{e}$ 09. Além de não esclarecer a dúvida levantada por sua irmã, abandona o subtópico e introduz outro (algo relacionado com Arturzinho no turno 10) ao qual C adere no turno 11. No trecho 2, a solicitação de esclarecimento feito por $\mathrm{C}$ no turno 02 só é esclarecida no turno 07 por IP (quando emite "isso"). IP parece apresentar dificuldade em manter a coerência tópica, na construção do esclarecimento solicitado (parece que não está compartilhando o assunto a ser esclarecido).

O Quadro 3, adaptado do manual de aplicação do CAPPCI, resume as habilidades e dificuldades de IP com relação ao gerenciamento do tópico, e seu impacto na conversação.

Quadro 2. Análise da iniciação e tomada de turno

\begin{tabular}{|lcc|}
\hline Área & Evidência na conversação & Gravidade do problema \\
\hline Habilidade em responder quando selecionado como próximo interlocutor & 0 & 0 \\
Habilidade em produzir enunciados que não contenham muitas pausas no meio & 2 & 1 \\
dos turnos & & 1 \\
Habilidade em responder sem violar o turno do parceiro conversacional & 1 & 1 \\
Habilidade em responder sem que haja sobreposição de vozes & 1 & 1 \\
Habilidade em responder sem que a resposta seja muito extensa & 2 & 1 \\
\hline
\end{tabular}

Legenda: Evidência na conversação: 0 = não apresenta dificuldade; 1 = apresenta dificuldade ocasionalmente; 2 = apresenta dificuldade frequentemente. Gravidade do problema: 0 = não prejudica a conversação; 1 = prejudica a conversação; 2 = prejudica seriamente a conversação.

Quadro 3. Análise do gerenciamento do tópico

\begin{tabular}{|lcc|}
\hline Área & Evidência na conversação & Gravidade do problema \\
\hline Habilidade em orientar o parceiro conversacional para novos tópicos & 1 & 2 \\
Habilidade de manter tópico & 1 & 1 \\
Habilidade em reformular um tópico quando há quebra do saber compartilhado & 1 & 2 \\
\hline
\end{tabular}

Legenda: Evidência na conversação: 0 = não apresenta dificuldade; 1 = apresenta dificuldade ocasionalmente; 2 = apresenta dificuldade frequentemente. Gravidade do problema: 0 = não prejudica a conversação; 1 = prejudica a conversação; 2 = prejudica seriamente a conversação. 
Quadro 4. Análise das reformulações

\begin{tabular}{|lcc|}
\hline Área & Evidência na conversação & Gravidade do problema \\
\hline Hetero-reformulações: & & 0 \\
Habilidade em solicitar uma reformulação ao interlocutor & 1 & 2 \\
Habilidade em reformular um enunciado a partir de uma solicitação por parte do & \\
parceiro conversacional & 0 & 0 \\
Auto-reformulações & 2 & 1 \\
Habilidade em realizar reformulações dos próprios erros & & 0 \\
Ensaios de formulação & & \\
\hline
\end{tabular}

Legenda: Evidência na conversação: 0 = não apresenta dificuldade; 1 = apresenta dificuldade ocasionalmente; 2 = apresenta dificuldade frequentemente. Gravidade do problema: 0 = não prejudica a conversação; 1 = prejudica a conversação; 2 = prejudica seriamente a conversação.

\section{Reformulações}

As hetero-reformulações foram analisadas com relação aos dois interlocutores, ou seja, com relação à habilidade em solicitar, ao interlocutor, uma reformulação (habilidade em solicitar uma reformulação ao interlocutor) e com relação às solicitações de reformulação feitas pela irmã (habilidade em reformular um enunciado a partir de uma solicitação por parte do PC). O exame da primeira habilidade revela que IP não solicitou, em nenhum momento, uma reformulação. Demonstra, portanto, que não apresenta dificuldades de compreensão no trecho analisado. Assim como já foi descrito acima, IP apresenta dificuldade na segunda habilidade. De fato, quando C solicita uma reformulação, IP apresenta dificuldade em resolvê-la prontamente (vide turno 07 a 10 do trecho 1 e turnos 02 a 07 do trecho 2).

Com relação às auto-reformulações (habilidade em realizar reformulações dos próprios erros), verificou-se que, nos dez minutos de conversação transcritos, IP realizou apenas um erro propriamente dito. Este foi de origem fonológica e foi imediatamente "reparado" com sucesso (emitiu "benco" ao invés de "banco").

A atenção de IP sempre parece estar dirigida "à parte seguinte do enunciado" em um mecanismo de busca prospectiva, o que leva a uma grande incidência de "ensaios de formulação" caracterizados por pausas de extensão variada, prolongamentos e repetições. Parece se dar conta dos problemas de formulação antes de formulá-los. Além da presença elevada de pausas, o discurso de IP apresenta muitas repetições de segmentos de palavra, palavras e segmentos de enunciado. No segundo turno do trecho 1, por exemplo, IP repete "antes eu não/ antigamente eu não" e "na ca/ na casa", além da repetição de pronomes, artigos e preposições. É interessante notar que, freqüentemente, começa um enunciado mas, por algum motivo, prefere reformular este enunciado, como se a primeira opção de formulação implicasse em dificuldades no processo formulativo. No segundo turno do trecho 1, por exemplo, muda a trajetória de sua formulação resolvendo emitir pela afirmativa um enunciado que ele havia iniciado na negativa ("eu num tinha tanto medo de sair (.) à noite (.) quanto eu tenho agora (1s.27ms.) porque:: nunca é::: muito mais (.) é muito maior o índice de de de violência”).

Apesar das inúmeras interrupções do fluxo formulativo em andamento, IP consegue encontrar alternativas de formulação e desenvolve segmentos complexos do ponto de vista sintático, com enunciados coordenados e subordinados, como pode ser observado no trecho abaixo (trecho 3 ).

\section{Trecho 3}

01. C - é que uma vez fiquei com impressão que tinham te assaltado de bicicleta?

02. IP - não não (41 ms.) nunca fizeram (40ms.) por isso que eu não/ por isso que e nem esquentava assim (27ms.) de sair de passeá:: tal (1s.27ms.) de saí de noite $(23 \mathrm{~ms}$.) porque nunca (43ms.) nunca tinha acontecido nada comigo de a/ de assalta:: de alguém chegar em mim (1s.49ms.) só dessa vez que:: (1s.42ms.) que:: e me pegaram $(27 \mathrm{~ms}$.) com o carro.

O Quadro 4 resume as habilidades e dificuldades de IP com relação às habilidades de formulação, e seu impacto na conversação.

\section{DISCUSSÃO}

O estudo dos três aspectos selecionados (iniciação e tomada de turno, gerenciamento do tópico e reformulações) para analisar a conversação forneceu elementos importantes para o processo de processo de reabilitação.

O estudo das pausas e repetições mostrou que, apesar de serem fontes potenciais de interrupção, seu uso parece ser uma estratégia positiva para que IP possa organizar e desenvolver seu fluxo formulativo. Sua irmã é uma interlocutora que respeita a maioria dessas pausas. Esta é, provavelmente, uma estratégia que ela desenvolveu para lidar com o impacto das dificuldades de formulação na conversação. Em outras palavras, a descontinuidade sintática não provoca a ruptura discursiva ${ }^{(10)}$.

O estudo da distribuição dos turnos revelou que, em vários momentos, houve sobreposição de vozes e interrupção do turno. Há necessidade de esclarecer os fatores que podem desencadear estas violações de turno e suas conseqüências para o desenvolvimento da interação. Além disso, em vários momentos, IP não percebe que não contribui de forma efetiva na resolução dos esclarecimentos solicitados por sua irmã, o que leva a uma fragmentação do discurso. Esses dados indicam a necessidade de desenvolver, no processo de reabilitação, a organização do discurso, focalizando não apenas o desenvolvimento do núcleo semântico-pragmático, mas também a consciência dessas dificuldades, pois, com muita freqüência, 
a própria natureza da lesão cerebral no TCE acaba limitando a capacidade do sujeito perceber suas dificuldades e em ajustar-se às mesmas. Seria interessante inserir, nos objetivos do processo de reabilitação, atividades metalingüísticas por meio das quais, por exemplo, o papel do "conhecimento compartilhado" seria discutido e pouco a pouco monitorado por meio de atividades dialógicas.

Quanto ao estudo das reformulações, é interessante notar que IP praticamente não comete erros, mas está, sempre, buscando alternativas de formulação que lhe permitem resolver impasses, garantindo, de certa forma, o desenvolvimento da interação. Do ponto de vista do processo de reabilitação, seria interessante discutir com ele, a partir da gravação de sua fala (portanto, por meio do controle executivo), as estratégias que ele espontaneamente desenvolve (pausas, reformulações...) e a necessidade das mesmas.

É interessante notar o quanto este estudo permite, constantemente, vislumbrar a relação entre controle executivo e conversação, tanto no que tange à organização da memória (guiando o processamento cognitivo pela representação mental ou simbólica - organização semântica), planejamento de resolução de problemas (habilidade em prever sub-objetivos e monitorar sua realização), e auto-regulação das respostas (iniciação, monitoração, inibição e revisão das respostas) por meio de atividades meta-lingüísticas.

\section{COMENTÁRIOS FINAIS}

As consequiências sociais das alterações da função comu- nicativa dos sujeitos com TCE, no longo-prazo, apontam para a necessidade de desenvolver instrumentos de avaliação que possam se adequar às particularidades do TCE.

Os resultados deste estudo indicam que a AC facilitou a identificação das alterações da função comunicativa, indo ao encontro das queixas de IP e das dificuldades que ele relata no âmbito profissional. Evidenciou dificuldades que não haviam sido capturadas nos testes formais, confirmando os achados de outro estudo ${ }^{(9)}$ acerca da validade da Análise Conversacional no estudo do TCE. Fornece, portanto, elementos concretos e próximos de suas dificuldades comunicativas cotidianas para que estes sejam discutidos com ele e incluídos no processo de reabilitação.

Além de propor um instrumento de avaliação, este estudo fornece informações importantes para a intervenção fonoaudiológica. Permite abordar de forma organizada a aparente "desorganização" da conversação, fornecendo elementos concretos para o processo de reabilitação. Permite discutir, a partir de uma análise do vídeo ou da transcrição da interação, portanto num contexto ecológico, as dificuldades reais do processo comunicativo, apresentadas por IP com sua irmã. Vislumbramos também a possível contribuição dessa abordagem no trabalho de re-inserção sócio-profissional dos sujeitos com TCE, analisando, por exemplo, sua interação com interlocutores menos familiares.

Apontamos, ainda, para a contribuição da AC na compreensão da interface entre discurso e habilidades metalingüísticas e na valorização do papel do controle executivo como "salvaguarda" da produção verbal.

\begin{abstract}
The persistence of communication impairments in individuals who sustained a traumatic brain injury requires the use of sensitive tools to evaluate the linguistic-cognitive problems they present, aiming at their reintegration in society. This single case study investigates the contributions of Conversation Analysis to the evaluation of the pragmatic abilities of a 27 year-old man who sustained a severe traumatic brain injury. It analyses a spontaneous conversational interaction through the study of the collaborative mechanism of turntaking, topic management and repairs, comparing the results of this analysis with formal language tests. It discusses the contributions of this approach to develop intervention. While formal tests did not indicate the presence of language difficulties, the Conversation Analysis allowed, through the analysis of the sequence of turns and of the mutual reactions of both the interlocutors, the identification of communicative problems and the observation of how the speakers dealt with them. The results of this study revealed that Conversation Analysis is a sensitive approach to capture the linguistic-cognitive deficits caused by traumatic brain injury, providing concrete elements to be used in therapy in order to emphasize, in an organized way, the kind of discourse most used in daily life.
\end{abstract}

KEYWORDS: Brain injury; Communication disorders; Language tests; Rehabilitation; Speech-language pathology; Case reports [Publication type]

\title{
REFERÊNCIAS
}

1. Galski T, Tompkins C, Johnston MV. Competence in discourse as a measure of social integration and quality of life in persons with traumatic brain injury. Brain Inj. 1998;12(9):769-82.

2. Penn C. Pragmatic assessment and therapy for persons with brain damage: what have clinicians gleaned in two decades? Brain Lang. 1999;68(3):535-52.
3. Body R, Perkins M, McDonald S. Pragmatics, cognition and communication in traumatic brain injury. In: McDonald S, Togher L, Code C, editors. Communication disorders following traumatic brain injury. East Sussex: Psychology Press; 1999. p. 81-112.

4. Steiner VAG, Mansur LL. Análise conversacional em indivíduos com traumatismo cranioencefálico. No prelo 2008. 
5. Goodwin C. Co-constructing meaning in conversations with an aphasic man. Res Lang Soc Interaction. 1995;28(3):233-60.

6. Wilkinson R. Introduction to 'Special Issue on Conversation Analysis'. Aphasiology. 1999;13(4):251-8.

7. Whitworth A, Perkins L, Lesser R. Conversation Analysis Profile for People with Aphasia (CAPPA). London: Whurr; 1997.

8. Whitworth A, Lesser R, McKeith I. Profiling conversation in Parkinson's disease with cognitive impairment. Aphasiology. 1999;13(4/5):407-25.

9. Friedland D, Miller N. Conversation analysis of communication breakdown after closed head injury. Brain Inj. 1998;12(1):1-14.

10. Marcuschi LA. A hesitação. In: Neves MHM, organizador. Gramática do português falado. São Paulo: Humanitas / Editora da Unicamp; 1999. p.159-94.[Novos Estudos, VII].

11. Schegloff EA. Repair after next turn: The last structurally provided defense of intersubjectivity in conversation. Am J Sociol. 1992;97(5):1295-345
12. Brito GN, Brito LS, Paumgartten FJ, Lins MF. Lateral preferences in Brazilian adults: an analysis with the Edinburgh Inventory. Cortex. 1989;25(3):403-15.

13. Radanovic M, Mansur LL. Performance of a Brazilian population sample in the Boston Diagnostic Aphasia Examination: a pilot study. Braz J Med Biol Res. 2002;35(3):305-17.

14. Mansur LL, Radanovic M, Araujo GC, Taquemori LY, Grecco LL. Teste de nomeação de Boston: desempenho de uma população de São Paulo. Pro-Fono. 2006;18(1):13-20.

15. De Renzi E, Vignolo LA. The Token test: A sensitive test to detect receptive disturbances in aphasics. Brain. 1962;85:665-78.

16. Schlenck KJ, Huber W, Willmes K. "Prepairs" and repairs: different monitoring functions in aphasic language production. Brain Lang. 1987;30(2):226-44

Apêndice. Símbolos da transcrição

01 - Número do turno.

IP - Inicial do interlocutor, no início de cada turno.

[ - Sobreposição de vozes.

\# - Interrupção.

(1s. 03ms.) - Tempo exato de duração da pausa (1 segundo e 3 milisegundos).

(.) - Pausa de duração aproximada de um décimo de segundo.

: - Prolongamento do som ou da sílaba (quanto maior o prolongamento, maior o número de símbolos).

/ - Corte abrupto de uma palavra ou de parte de uma palavra. 